\title{
Effect of Toothbrushing on Surface Color of Ceramic-polymer Materials: An In Vitro Study
}

\author{
Farzan L Pouranfar ${ }^{1}$, Ryan Sheridan ${ }^{2}$, Cade Salmon ${ }^{3}$, Kraig S Vandewalle ${ }^{4}$
}

\begin{abstract}
Aim and objective: The purpose of this study was to examine the effects of toothbrushing on the change in color of extrinsic characterization of ceramic-polymer materials.

Materials and methods: Two ceramic-polymer materials (CeraSmart, GC; Enamic, VITA) and one lithium-disilicate material (IPS e.max CAD; Ivoclar Vivadent) were tested. Specimens of each material were prepared, characterized, and glazed per manufacturer's instructions. The treated surface of the blocks were then brushed in a toothpaste slurry with artificial saliva using a toothbrush machine with a soft toothbrush. Commission Internationale de L'Eclairage (CIE) $L^{*} a^{*} b^{*}$ values were recorded with a spectrophotometer at baseline and at 3,6, 9, and 12 simulated years of brushing (7,300 strokes/year). A mean change in color $\left(\Delta E^{*}\right)$ and standard deviation was determined for each group and brushing interval. Data were analyzed with a two-way repeated measures ANOVA examining the effects of toothbrushing the ceramic materials on $\Delta E^{*}$ over time $(\alpha=0.05)$. Results: The difference in the $\Delta E^{*}$ between CeraSmart and Enamic was significant at 3 years, while the differences between them were not significant at 6,9 , and 12 years of simulated brushing. The $\Delta E^{*}$ of IPS e.max CAD was significantly lower than CeraSmart and Enamic at all time points (all $p<0.0001$ ) except for the comparison with Enamic at 3 years.

Conclusion: The extrinsic stains on the ceramic-polymer materials may be more susceptible to change from simulated toothbrushing compared to the lithium-disilicate material.

Clinical significance: Toothbrushing may change the color of extrinsic characterization of ceramic-polymer materials. However, the change may remain clinically imperceptible to the naked eye $\left(\Delta E^{*}>1.0\right)$ for nearly 6 equivalent years of brushing.

Keywords: Ceramic polymers, Extrinsic stains, Lithium disilicate, Surface color, Toothbrushing.

The Journal of Contemporary Dental Practice (2020): 10.5005/jp-journals-10024-2933
\end{abstract}

\section{INTRODUCTION}

Ceramic restorations are usually characterized via superficial application of pigments (commonly known as "extrinsic staining"). These pigments are strategically applied using a fine brush to create desired effects, colors, and shades..$^{1-3}$ Unlike intrinsic characterization of ceramic restorations, extrinsic characterization is in direct contact with the oral environment and abrasives, and therefore at higher risk of fading. ${ }^{3}$ Consequently, it has been recommended that extrinsic stains be placed as deeply as possible to enhance longevity. ${ }^{4}$

Toothbrushing with fluoride-containing toothpaste is the most effective approach for plaque removal and dental-caries prevention. ${ }^{5-7}$ Unfortunately, abrasives are an integral component of toothpastes and may have an adverse effect on surface characterization of ceramic restorations. These effects could vary depending on the type of the restorative material. There are three main groups of dental ceramic and ceramic-like materials available-glass-matrix, polycrystalline, and ceramic polymers. ${ }^{8}$ Glass-matrix ceramics contain nonmetallic inorganic ceramic materials in a glass phase and are the most esthetic. Examples include feldspathic ceramics, leucite-based ceramics, and lithium disilicate and derivatives. ${ }^{9}$ Polycrystalline ceramics like alumina and stabilized zirconia contain nonmetallic inorganic ceramic materials but do not present any glass phase and may therefore be less esthetic without extrinsic staining or esthetic ceramic veneering. Ceramic polymers are made up of predominantly inorganic refractory compounds that usually include porcelains, glasses, ceramics, glass ceramics, in addition to organic polymers, but may require extrinsic staining to achieve acceptable clinical esthetics. ${ }^{8}$
${ }^{1}$ Department of Clinical Dentistry, Dental Clinic, Ft. Polk, Louisiana, USA

2,3 Department of Prosthodontics, Prosthodontic Residency, Air Force Postgraduate Dental School, Joint Base San Antonio-Lackland, Texas, USA; Uniformed Services, University of the Health Sciences, Bethesda, Maryland, USA

${ }^{4}$ Department of General Dentistry, Advanced Education in General Dentistry Residency, Joint Base San Antonio-Lackland, Texas, USA Uniformed Services, University of the Health Sciences, Bethesda, Maryland, USA

Corresponding Author: Kraig S Vandewalle, Department of General Dentistry, Advanced Education in General Dentistry Residency, Joint Base San Antonio-Lackland, Texas, USA; Uniformed Services, University of the Health Sciences, Bethesda, Maryland, USA, Phone: +1 210-292-0760, e-mail: kraig.s.vandewalle.civ@mail.mil

How to cite this article: Pouranfar FL, Sheridan R, Salmon C, et al. Effect of Toothbrushing on Surface Color of Ceramic-polymer Materials: An In Vitro Study. J Contemp Dent Pract 2020;21(9):1054-1058.

Source of support: 59th Clinical Investigations and Research Support, Joint Base San Antonio-Lackland, TX.

Conflict of interest: None

These new ceramic-polymer materials appear to fall on a spectrum between polymer and ceramic, with some materials, such as Lava Ultimate (3M ESPE, St. Paul, MN, USA), CeraSmart (GC, Tokyo, Japan), and Shofu HC (Shofu, Kyoto, Japan) containing primarily polymer materials. On the other hand, Enamic (VITA, Bad Sackingen, Germany) reportedly contains more ceramic material. ${ }^{8,10,11}$ The two ceramic-polymer materials, CeraSmart and

() The Author(s). 2020 Open Access This article is distributed under the terms of the Creative Commons Attribution 4.0 International License (https://creativecommons. org/licenses/by-nc/4.0/), which permits unrestricted use, distribution, and non-commercial reproduction in any medium, provided you give appropriate credit to the original author(s) and the source, provide a link to the Creative Commons license, and indicate if changes were made. The Creative Commons Public Domain Dedication waiver (http://creativecommons.org/publicdomain/zero/1.0/) applies to the data made available in this article, unless otherwise stated. 
Enamic, represent both ends of the spectrum of ceramic-polymer materials and are marketed with a dedicated proprietary lightpolymerized staining kit.

Extrinsic characterization of dental ceramics is usually carried out by applying a desired colorant to external surfaces of a restoration to achieve the desired effect. According to Ivoclar Vivadent (Schaan, Liechtenstein) technical instructions for lithium-disilicate glass ceramics such as IPS e.max CAD, the characterized restoration must be placed in a ceramic oven capable of reaching temperatures of $840^{\circ} \mathrm{C}^{12}$ In contrast, manufacturers recommend extrinsic characterization of ceramic polymers via visible-light polymerization of their methacrylate-based surface stains. ${ }^{13,14}$ However, the longevity of these visible light-activated surface stains has not been validated by clinical studies. The purpose of this in vitro study was to evaluate the effect of toothbrushing on the surface of ceramic-polymer materials, specifically surfaces treated with visible light-cured stains. The null hypothesis suggested there would be no difference in the change in color based on the type of ceramic material over 3, 6, 9, and 12 equivalent years of simulated toothbrushing.

\section{Materials and Methods}

The study was conducted in the United States Air Force Postgraduate Dental School at Joint Base San Antonio, Lackland, TX, USA. The following materials were tested: two ceramic-polymersEnamic (VITA) and CeraSmart (GC) and one lithium disilicate glassceramic - IPS e.max CAD (Ivoclar Vivadent). Enamic is composed of predominately a feldspar-ceramic network enriched with aluminum oxide $(86 \%$ wt or $75 \%$ vol) and a polymethylmethacrylate $(14 \% \mathrm{wt}$ or $25 \%$ vol) that permeates the feldspar-ceramic matrix to yield a restorative material that is both strong and elastic as claimed by the manufacturer. ${ }^{11}$ CeraSmart is composed of a combination of bis-methacryloxyethoxy phenyl propane (BisMEPP), urethane dimethacrylate (UDMA), and dimethacrylate (DMA) with silica and barium glass fillers at $71 \%$ by weight. ${ }^{15}$ The glass-ceramic, lithium disilicate (IPS e.max CAD, Ivoclar Vivadent) was chosen as the control because it is a commonly used material that has been well studied and clinically successful with over $96 \%$ success rate. ${ }^{16,17}$ The material is milled at an intermediate blue state composed of $40 \%$ lithiummetasilicate crystals. After crystallization, the material crystallizes to approximately $70 \%$ fine-grain lithium disilicate, creating increased strength and wear resistance. ${ }^{12}$

Ten blocks (size C14) from each of the three tested materials $(n=10)$ in shade A2 were polished using a lapping table (Ecomet 6, Buehler, Lake Bluff, IL, USA) in conjunction with silicon-carbide paper, which ranged from 320 grit through 420 grit. A 3-D printed index (Eden 260V 3-D printer, Stratasys, Eden Prairie, MN, USA) was utilized to hold each block specimen in the lapping table (Figs $1 \mathrm{~A}$ and B). After polishing, each block's thickness was measured with a digital caliper (GA182, Grobet Vigor, Carlstadt, NJ, USA).

Enamic specimens were treated with the Enamic Stain Kit (VITA) according to manufacturer's instructions. ${ }^{13}$ The surface was etched with a $5 \%$ hydrofluoric acid gel (Ceramics Etch, VITA) for 60 seconds and then steam cleaned (i700B, Reliable, Toronto, Ontario, Canada). The cleaning solution included in the kit (Cleaner, VITA) was applied and air-dried. Then the block was covered with 120- $\mu$ m-thick tape (Masking Tape, 3M, Maplewood, MN, USA) with a window cut in the area to standardize the thickness of the stain and glaze (Fig. 1C). Next, silane (VitaSil, VITA) was applied and airdried. Stain 3 Brown was applied and light-cured for 30 seconds with Bluephase G2 LED Curing Light (Ivoclar Vivadent) in the "high" setting. Irradiance of the curing light was monitored with a radiometer (LED Radiometer, Kerr, Orange, CA, USA) to verify irradiance levels above $1,200 \mathrm{~mW} / \mathrm{cm}^{2}$. Glaze was applied with a clean disposable brush and light polymerized for an additional 60 seconds. Using the digital caliper, the thickness of the stain and glaze was measured to assure standardization. The stain and the glaze were each approximately $60 \mu \mathrm{m}$ thick for each block.

CeraSmart blocks were stained and glazed using Optiglaze (GC) according to the manufacturer's instructions. ${ }^{14}$ First, the CeraSmart blocks were sandblasted (AccuFlo, Comco Inc., Burbank, CA, USA) with $50 \mu \mathrm{m}$ of aluminum oxide at a distance of $10 \mathrm{~mm}$ at $80 \mathrm{PSI}$ and steam cleaned. Then, the Optiglaze Ceramic Primer II was applied and air-dried. Masking tape was used in the same manner described above for standardization of the extrinsic stain and glaze. Stain A-plus brown was applied and light-cured for 40 seconds with the curing light as before. Finally, Optiglaze Clear was applied and light-cured for 60 seconds.

For the IPS e.max CAD blocks, the metal stubs were removed and the blocks were crystallized within a ceramic oven (Programat P500, Ivoclar Vivadent) as per the manufacturer's recommendation (Fig. 1D). Masking tape was used in the same manner described above. Stain (IPS e.max Crystall./Shade 1, Ivoclar Vivadent) and glaze (IPS e.max Crystall./Glaze and Glaze Liquid, Ivoclar Vivadent) were placed within the taped area. The tape was removed and the block was placed back in the oven for the staining and glazing cycle according to the manufacturer's instructions. The metal stubs were then reattached (Fig. 1E) to the IPS e.max CAD blocks with a commercial adhesive (Gorilla Glue, Gorilla Glue Co., Cincinnati, $\mathrm{OH}, \mathrm{USA})$.

Baseline surface color measurements for individual block specimens (CIE- $\left.L^{*} a^{*} b^{*}\right)$ were recorded with the spectrophotometer (Easyshade, VITA). A 3-D printed index (Eden 260V 3-D printer, Stratasys) was utilized to hold each block specimen and the spectrophotometer in the same orientation every time the color was measured (Fig. 1F).

The 10 specimens per group were mounted in a 10-station automated toothbrush machine (Sabri Dental Enterprises, Downers Grove, IL, USA) using 3-D printed indexes (Fig. 1G). Soft filament toothbrush heads were sectioned from brush handles (GUM 311 Classic Toothbrush, Sunstar, Schaumburg, IL, USA) and fixed to the machine brush arm. Each toothbrush head was applied to the stained surfaces with a force of $3 \mathrm{~N}$. All specimens were brushed for 1 -year intervals up to 12 equivalent years of brushing at a rate of 60 strokes per minute $(1 \mathrm{~Hz})$. Toothbrush heads were replaced every 7,300 strokes (1-year equivalent). ${ }^{18}$ An abrasive slurry consisting of toothpaste (Crest, Procter and Gamble, Cincinnati, $\mathrm{OH}$ ) at a mixture of $21 \mathrm{~g}$ per $80 \mathrm{~mL}$ of synthetic mouth fluid was utilized for experimental purposes. The synthetic saliva was prepared with the following solutions: $\mathrm{Na}_{3} \mathrm{PO}_{4}-3.90 \mathrm{mM}, \mathrm{NaCl}_{2}-4.29 \mathrm{mM}$, $\mathrm{KCl}-17.98 \mathrm{mM}, \mathrm{CaCl}_{2}-1.10 \mathrm{mM}, \mathrm{MgCl}_{2}-0.08 \mathrm{mM}, \mathrm{H}_{2} \mathrm{SO}_{4}-0.50$ $\mathrm{mM}, \mathrm{NaHCO}_{3}-3.27 \mathrm{mM}$, distilled water, with a $\mathrm{pH}$ set to a level of 7.2. ${ }^{19} \mathrm{~A} \mathrm{pH}$ meter (Accumet XL50, Fisher Scientific, Waltham, $\mathrm{MA}$ ) was used to measure $\mathrm{pH}$. Specimens were removed from the automated toothbrush machine (Fig. $1 \mathrm{H}$ ) at prescribed intervals (i.e., $3,6,9$, and 12 equivalent years of simulated brushing), and color measurements were performed using the spectrophotometer and 3-D printed index to orient each sample in the same way to the spectrophotometer tip. Changes in color of the ceramic-polymer materials from the toothbrushing process were determined using the CIE $L^{*} a^{*} b^{*}$ color space. $L^{*}$ indicates lightness $(L+=$ lightness and $\mathrm{L}-=$ darkness), the $\mathrm{a}^{*}$ coordinate represents the red/green range $\left(a^{*}+=\right.$ redness and $a^{*}-=$ greenness), and the $b^{*}$ coordinate 

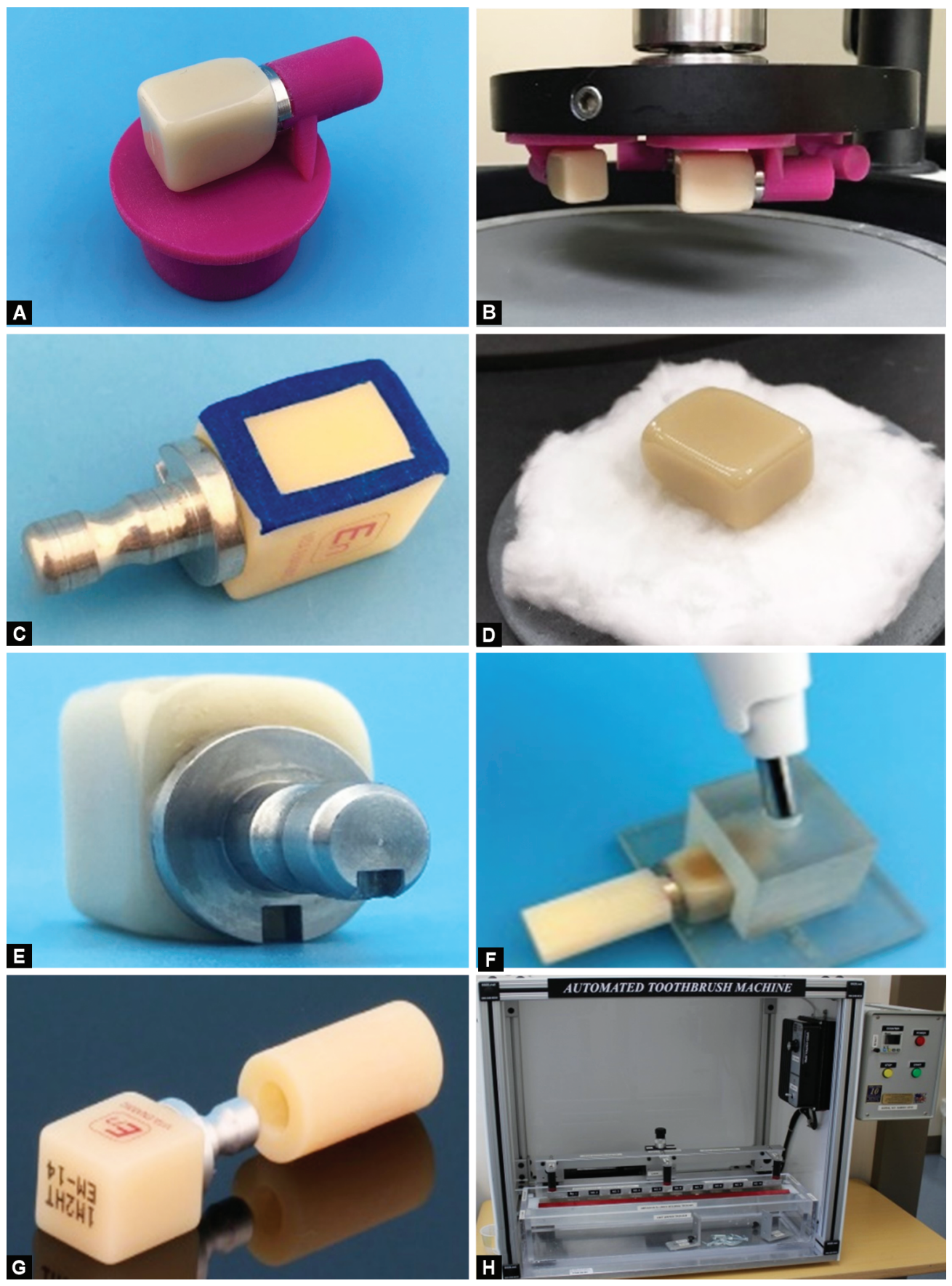

Figs $1 \mathrm{~A}$ to H: (A) Custom index for polishing the blocks; (B) Specimen polished in automated lapping table; (C) Tape placement for standardization of surface stains; (D) Crystallized IPS e.max CAD block; (E) Reattachment of stub on IPS e.max CAD; (F) Custom index for color measurement; (G) Custom index for brushing; $(\mathrm{H})$ Ten-specimen custom toothbrushing machine

represents for the yellow/blue range $\left(b^{*}+=\right.$ yellowness and $b^{*}-=$ blueness). The $L^{*} a^{*} b^{*}$ system allows the numeric definition of a color as well as the overall difference between two colors $\left(\Delta E^{*}\right){ }^{20}$ The mean of three readings per specimen was recorded for each $\mathrm{L}^{*} \mathrm{a}^{*} \mathrm{~b}^{*}$ value and standard deviations were determined for each group. Color change was measured using the formula: $\Delta E^{*}=$ $\left[\left(\Delta L^{*}\right)^{2}+\left(\Delta a^{*}\right)^{2}+\left(\Delta b^{*}\right)^{2}\right]^{1 / 2}$. Two-way repeated measures ANOVA was conducted to test the effects of material and time on $\Delta E^{*}$. The sample size of 10 specimens per group provided $80 \%$ power to detect a moderate effect size $(0.3$, or approximately 0.6 standard deviation difference) among means for the main factors of group and time, and a moderate effect $(0.35$, or approximately 0.7 standard deviation difference) for the interaction term when testing with a two-way repeated measures ANOVA at the alpha level of 0.05 (NCSS PASS 2002).

\section{Results}

After 12 years of simulated toothbrushing, CeraSmart had the greatest $\Delta E^{*}(3.3 \pm 0.2)$, but it was not significantly greater $(p=0.88)$ 
than Enamic ( $3.1 \pm 0.3)$. IPS e.max CAD had the lowest $\Delta E^{*}(0.8 \pm 0.2)$ after 12 years of simulated toothbrushing, and it was significantly less ( $p<0.0001$ ) than CeraSmart and Enamic (Table 1 and Fig. 2).

Two-way repeated measures ANOVA with a GreenhouseGeisser correction showed that overall main effects of material and time and the interaction effect between material and time were statistically significant: material $[\mathrm{F}(2,27)=359.72, p<0.0001]$; time $[F(2.31,62.33)=697.35, p<0.0001]$; material*time $[F(4.62,62.33)$ $=77.02, p<0.0001]$. The significant interaction between material and time indicates that the differences among the material groups varied at different time points. Post hoc tests using the Tukey correction for the material and time interaction indicated that the difference in the $\Delta E^{*}$ between Enamic and IPS e.max CAD was not significant $(p=0.46)$ at 3 years, while the differences between them were significant $(p<0.0001)$ at 6,9 , and 12 years of simulated brushing. On the other hand, the difference in the $\Delta E^{*}$ between CeraSmart and Enamic was significant $(p<0.0001)$ at 3 years, while the differences between them were not significant at 6 years ( $p=$ $0.94)$, 9 years $(p=1.00)$, and 12 years $(p=0.88)$ of simulated brushing. Except for the comparison with Enamic at 3 years, the $\Delta E^{*}$ of IPS e.max CAD was significantly smaller than CeraSmart and Enamic at all time points (all $p<0.0001$ ).

\section{Discussion}

Indirect ceramic-polymer materials are created under optimized temperature and pressure settings for a controlled and standardized

Table 1: Mean $\Delta E^{*}$ ( \pm one standard deviation) for each of the ceramic materials at each simulated time interval

\begin{tabular}{lllll}
\hline & \multicolumn{4}{c}{ Mean $\Delta E^{*}$ (st. dev.) } \\
\cline { 2 - 5 } Materials & 3years & 6years & 9years & 12 years \\
\hline CeraSmart & $0.7(0.1) \mathrm{Aa}$ & $1.2(0.1) \mathrm{Ba}$ & $1.8(0.1) \mathrm{Ca}$ & $3.3(0.2) \mathrm{Da}$ \\
(GC) & & & & \\
Enamic (Vita) & $0.4(0.04) \mathrm{Ab}$ & $1.1(0.2) \mathrm{Ba}$ & $1.9(0.4) \mathrm{Ca}$ & $3.1(0.3) \mathrm{Da}$ \\
IPS e.max & $0.2(0.1) \mathrm{Ab}$ & $0.2(0.1) \mathrm{Ab}$ & $0.3(0.1) \mathrm{Bb}$ & $0.8(0.2) \mathrm{Cb}$ \\
CAD (ivoclar & & & & \\
vivadent) & & & &
\end{tabular}

Groups with the same uppercase letter per row or lowercase letter by column are not significantly different $(p>0.05)$

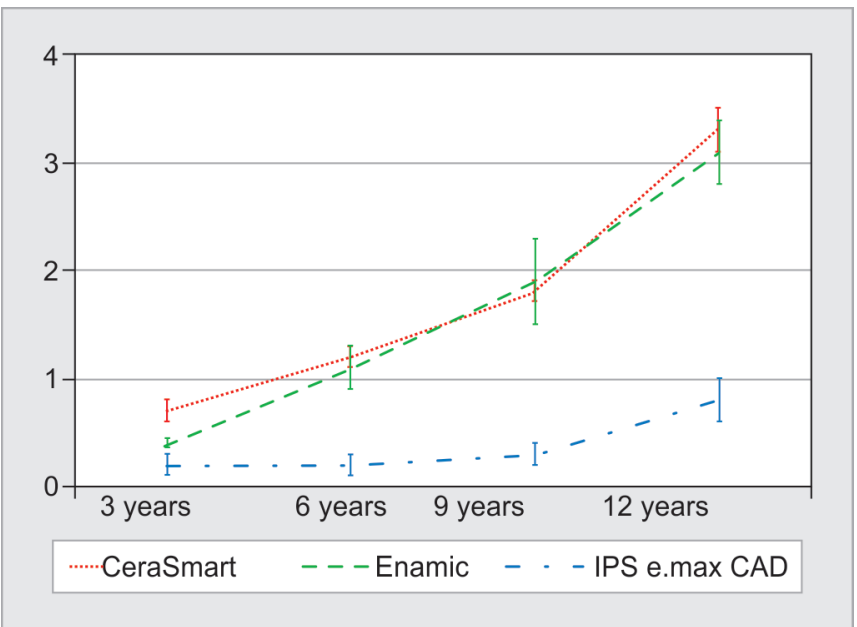

Fig. 2: Mean change in $\Delta E^{*}$ of surface stains after automated toothbrushing over time with the various ceramic materials polymerization. Polymerization in the laboratory allows for enhanced mechanical strength and improved stain resistance when compared to the direct resin composite material. ${ }^{21,22}$ Additionally, these polymers can be repaired using light-cured composite materials and readily characterized with light-polymerized stains. Extrinsic stains may be necessary to achieve comparable esthetics to ceramic materials. ${ }^{16}$ However, the newer ceramic-polymer materials may be less resistant to the abrasive effects of toothbrushing compared to ceramic materials.

In this study, significant increases in $\Delta E^{*}$ were observed in the two stained and glazed ceramic-polymer materials over a 12-year equivalent of simulated toothbrushing when compared to extrinsic stains on IPS e.max CAD. Therefore, the null hypothesis was rejected. Garza et al. showed that it required 12 years of simulated toothbrushing for noticeable change on extrinsic stains on IPS e.max Press, a pressable lithium-disilicate material. ${ }^{23}$ The current study found a minimal effect on IPS e.max CAD with only a $0.8 \pm$ 0.2 increase in $\Delta E^{*}$ after 12 years of simulated toothbrushing. $A E^{*}$ measure of 1.0 is the most frequently reported threshold value for the perceptibility of color differences. ${ }^{20}$ Compared to the IPS e.max CAD extrinsic stains, both CeraSmart and Enamic extrinsic stains displayed a statistically significant increase in $\Delta E^{*}$ starting at 6 years of simulated brushing. At that time, the surface changes with both CeraSmart and Enamic may be clinically noticeable with an increase of $\Delta E^{*}$ of $1.2 \pm 0.1$ and $1.1 \pm 0.2$, respectively. Conversely, the findings of the current study suggest that changes in the visible light-polymerized extrinsic stains of ceramic-polymer materials may remain imperceptible to the eye for at least 3 years of toothbrushing.

In a recent study by Partin-Agarwal et al., the surface roughness of new CAD/CAM ceramic-polymer materials without any surface stains or glazes was evaluated before and after abrasion with a toothbrush and dentifrice. Compared to the tested ceramic-polymer materials, only IPS e.max CAD had no statistically significant increase in surface roughness after 60,000 brush strokes (a simulated 8 years of toothbrush abrasion). ${ }^{24}$ This result is not unexpected, as lithiumdisilicate ceramic has been shown to exhibit high polishability and polish retention. ${ }^{25}$ In a similar study performed by Koizumi et al., both ceramic polymers tested demonstrated significantly rougher surfaces than a feldspathic porcelain (Vita Mark II, Vita) after toothbrush abrasion. ${ }^{26}$

The focus of the current study was to test extrinsic color longevity when normal toothbrushing was employed. Only two ceramic-polymer materials were evaluated in this study because currently very few companies of ceramic-polymer materials provide a proprietary stain and glaze kit. In addition, only one type of toothbrush and one type of toothpaste were used. There may be large variations in the amount of force that people use while brushing, the abrasiveness of the toothpaste, the softness of the toothbrush bristles, and the number of times per day individuals brush their teeth. Based on studies showing that humans have an average brushing force of 2-3 N, the toothbrush heads in this study were applied to the polished surface with a force of $3 \mathrm{~N}^{27}$ Manley determined the average daily brushing interval to be 19.72 strokes per surface per tooth resulting in the 7,300 strokes per simulated year used in this study. ${ }^{18}$ The literature reports conflicting information pertaining to the amount of brush strokes needed to simulate toothbrush abrasion. In various studies, 20,000 simulated toothbrush strokes were defined as the equivalent of 2 years or 7-10 years. ${ }^{28,29}$ Based on different definitions, the simulation of 87,600 toothbrush strokes on the glass-ceramic and ceramic-polymer 
materials in the present study may therefore replicate from 8.75 to 43.75 years of general toothbrushing wear. Furthermore, the impact of elements such as patient habits and diet must be further studied. Last, systemic diseases causing elevated gastric acid levels may negatively affect extrinsic stains and glazes. ${ }^{30}$ Therefore, additional research is needed to better understand clinical significance of surface color changes demonstrated by ceramic-polymer materials in association with the various factors described. Limitations to this study include the use of only two ceramic-polymer materials, one brand and type of toothbrush, and one type and level of brushing force.

\section{Conclusion}

Toothbrushing may change the color of extrinsic characterization of ceramic-polymer materials. However, the change may remain clinically imperceptible to the naked eye $\left(\Delta E^{*}>1.0\right)$ for nearly 6 equivalent years of brushing the ceramic-polymer materials.

\section{Disclaimer}

The views expressed are those of the authors and do not reflect the official views or policy of the Uniformed Services University, Department of Defense, or its components. The authors do not have any financial interest in the companies whose materials are discussed in this abstract study.

\section{References}

1. Bativala $F$, Weiner $S$, Berendsen $P$, et al. The microscopic appearance and effect of toothbrushing on extrinsically stained metal-ceramic restorations. J Prosthet Dent 1987;57(1):47-52. DOI: 10.1016/00223913(87)90115-6.

2. Lund TW, Schwabacher WB, Goodkind RJ. Spectrophotometric study of the relationship between body porcelain color and applied metallic oxide pigments. J Prosthet Dent 1985;53(6):790-796. DOI: 10.1016/0022-3913(85)90158-1.

3. Aker DA, Aker JR, Sorensen SE. Toothbrush abrasion of colorcorrective porcelain stains applied to porcelain-fused-to-metal restorations. J Prosthet Dent 1980;44(2):161-163. DOI: 10.1016/00223913(80)90130-4.

4. Anil N, Bolay S. Effect of toothbrushing on the material loss, roughness, and color of intrinsically and extrinsically stained porcelain used in metal-ceramic restorations: an in vitro study. Int J Prosthodont 2002;15(5):483-487.

5. Featherstone DB, Domejean-Orliaguet $\mathrm{S}$, Jenson $\mathrm{L}$, et al. Caries risk assessment in practice for age 6 through adult. J Calif Dent Assoc 2007;35(10):703-713.

6. Van der Weijden GA, Timmerman MF, Danser MM, et al. Relationship between the plaque removal efficacy of a manual toothbrush and brushing force. J Clin Periodontol 1998;25(5):413-416. DOI: 10.1111/j.1600-051x.1998.tb02464.x.

7. Arai T, Kinoshita S. A comparison of plaque removal by different toothbrushes and toothbrushing methods. Bull Tokyo Med Dent Univ 1977;24(2):177-188.

8. Gracis S, Thompson VP, Ferencz JL, et al. A new classification system for all-ceramic and ceramic-like restorative materials. Int J Prosthodont 2015;28(3):227-235. DOI: 10.11607/ijp.4244.

9. Nasr E, Makhlouf AC, Zebouni E, et al. All-ceramic computer-aided design and computer-aided manufacturing restorations: evolution of structures and criteria for clinical application. J Contemp Dent Pract 2019;20(4):516-523. DOI: 10.5005/jp-journals-10024-2549.

10. 3M ESPE. Lava Ultimate CAD/CAM Restorative. https://multimedia.3m. $\mathrm{com} / \mathrm{mws} / \mathrm{media} / 7561670 / 3 \mathrm{~m}$-lava-ultimate-cad-cam-restorativefor-cerec-the-edge-you-need.pdf Accessed April 14, 2020.
11. VITA Enamic [technical and scientific documentation]. https:// cdn.vivarep.com/contrib/vivarep/media/pdf/4_4642_ ENAMICTechnicalandScientific_20170830205744578.pdf. February 2018. Accessed April 14, 2020.

12. Ivoclar Vivadent technical, version 7 2017. http://www. ivoclarvivadent.com/zoolu-website/media/document/4611/Progr amat+P300\%2C+P500\%2C+P700\%2C+EP+3000\%2C+EP+5000++Firing+program+tables.pdf. Accessed April 14, 2020.

13. VITA Enamic Bonding Protocol. https://cdn. vivarep.com/contrib/vivarep/media/pdf/4_4643_ VITAENAMICBondingandPolishingGuide_20170830205802515.pdf. Accessed April 14, 2020.

14. GC, Optiglaze color instructions for use: http://www.gcamerica.com/ products/digital/OPTIGLAZE_color/OPTIGLAZE-color-IFU12L.pdf. Accessed April 14, 2020.

15. GC America. CeraSmart [instructions for use]. June 2016. http://www. gcamerica.com/products/digital/CERASMART/CERASMART_12IFU. pdf. Accessed April 14, 2020.

16. Fasbinder DJ, Neiva GF, Dennison JB, et al. Clinical performance of CAD/CAM-generated composite inlays after 10 years. J Cosmet Dent 2013;28:134-145.

17. Sailer I, Makarov NA, Thoma DS, et al. All-ceramic or metal ceramic tooth-supported fixed dental prostheses (FDPs)? A systemic review of the survival and complication rates. Part I: single crowns (SCs). Dent Mater 2015 31(6):603-623. DOI: 10.1016/j.dental.2015.02.011.

18. Manly RS. The abrasion of cementum and dentin by modern dentifrices. J Dent Res 1941;20(6):583-595. DOI: 10.1177/00220345410200060901.

19. Lata S, Varghese NO, Varughese JM. Remineralization potential of fluoride and amorphous calcium phosphate-casein phosphor peptide on enamel lesions: an in-vitro comparative evaluation. J Conserv Dent 2010;13(1):42-46. DOI: 10.4103/0972-0707.62634.

20. Khashayar G, Bain PA, Salari S, et al. Perceptibility and acceptability thresholds for colour differences in dentistry. J Dent 2014;42(6):637644. DOI: 10.1016/j.jdent.2013.11.017.

21. Guth JF, Zuch T, Zwinge S, et al. Optical properties of manually and CAD/CAM-fabricated polymers. Dent Mater J 2013;32(6):865-871. DOI: 10.4012/dmj.2013-099.

22. Aroucha MA, Basilio J, Llopis J, et al. Colour stainability of indirect CAD-CAM processed composites vs. conventionally laboratory processed composites after immersion in staining solutions. J Dent 2014;42(7):831-838. DOI: 10.1016/j.jdent.2014.04.002.

23. Garza LA, Thompson G, Cho SH, et al. Effect of toothbrushing on shade and surface roughness on extrinsically stained pressable ceramics. J Prosthet Dent 2016;115(4):489-494. DOI: 10.1016/j. prosdent.2015.09.013.

24. Partin-Agarwal K, Terwilliger RJ, Lien W, et al. Polish retention of ceramic-polymer CAD/CAM materials. Gen Dent 2018;66(6):65-70.

25. Mörmann WH, Stawarczyk B, Ender A, et al. Wear characteristics of current aesthetic dental restorative CAD/CAM materials: two-body wear, gloss retention, roughness and martens hardness. J Mech Behav Biomed Mater 2013;20:113-125. DOI: 10.1016/j.jmbbm.2013. 01.003.

26. Koizumi H, Saiki O, Nogawa $H$, et al. Surface roughness and gloss of current CAD/CAM resin composites before and after toothbrush abrasion. Dent Mater J 2015;34(6):881-887. DOI: 10.4012/dmj.2015-177.

27. Litonjua LA, Andreana S, Bush PJ, et al. Wedged cervical lesions produced by toothbrushing. Am J Dent 2004;17(4):237-240.

28. Tanoue N, Matsumura H, Atsuta M. Analysis of composite type and different sources of polymerization light on in vitro toothbrush/ dentifrice abrasion resistance. J Dent 2000;28(5):355-359. DOI: 10.1016/s0300-5712(00)00014-2.

29. Saxer UP, Barbakow J, Yankell SL. New studies on estimated and actual toothbrushing times and dentifrice use. J Clin Dent 1998;9(2):49-51.

30. Kulkarni A, Rothrock J, Thompson J. Impact of gastric acid induced surface changes on mechanical behavior and optical characteristics of dental ceramics. J Prosthodont 2020;29(3):207-218. DOI: 10.1111/ jopr.12716. 